\title{
Corrigendum
}

\section{Corrigendum to "Segmentation of Hyperacute Cerebral Infarcts Based on Sparse Representation of Diffusion Weighted Imaging"}

\author{
Xiaodong Zhang, ${ }^{1,2}$ Shasha Jing, ${ }^{1}$ Peiyi Gao, ${ }^{3}$ Jing Xue, ${ }^{3}$ Lu Su, ${ }^{3}$ Weiping Li, ${ }^{4}$ Lijie Ren, ${ }^{4}$ \\ and Qingmao $\mathrm{Hu} \mathbb{1}^{1,2}$ \\ ${ }^{1}$ Shenzhen Institutes of Advanced Technology, Chinese Academy of Sciences, 1068 Xueyuan Boulevard, Shenzhen 518055, China \\ ${ }^{2}$ University of Chinese Academy of Sciences, Beijing, China \\ ${ }^{3}$ Beijing Tiantan Hospital, Capital Medical University, 6 Tiantan Xili, Beijing 100050, China \\ ${ }^{4}$ Shenzhen Second People's Hospital, 3002 West Sungang Road, Shenzhen 518035, China \\ Correspondence should be addressed to Qingmao Hu; qm.hu@siat.ac.cn
}

Received 11 October 2018; Accepted 16 October 2018; Published 5 November 2018

Copyright (c) 2018 Xiaodong Zhang et al. This is an open access article distributed under the Creative Commons Attribution License, which permits unrestricted use, distribution, and reproduction in any medium, provided the original work is properly cited.

In the article titled "Segmentation of Hyperacute Cerebral Infarcts Based on Sparse Representation of Diffusion Weighted Imaging" [1], there was a missing affiliation for the first and last authors. The corrected authors' list and affiliations are shown above.

\section{References}

[1] X. Zhang, S. Jing, P. Gao et al., "Segmentation of hyperacute cerebral infarcts based on sparse representation of diffusion weighted imaging," Computational and Mathematical Methods in Medicine, vol. 2016, Article ID 2581676, 14 pages, 2016. 


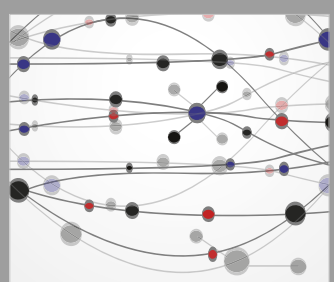

The Scientific World Journal
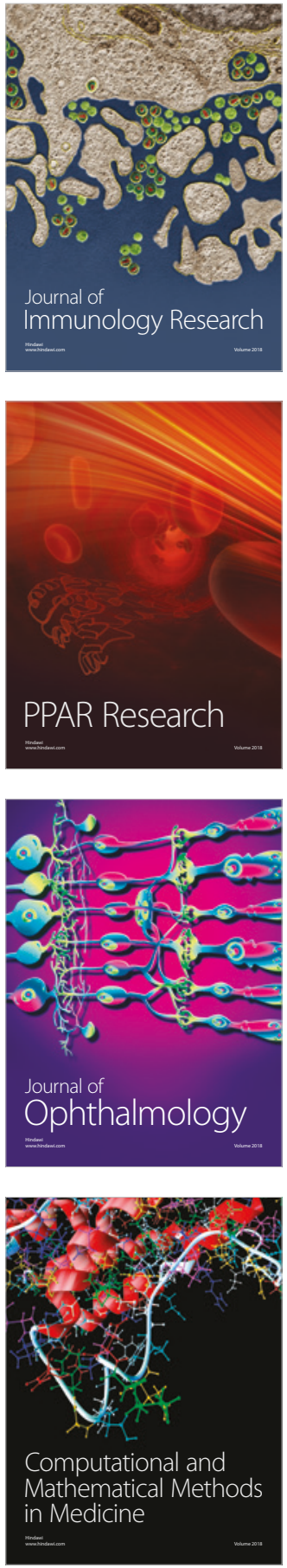

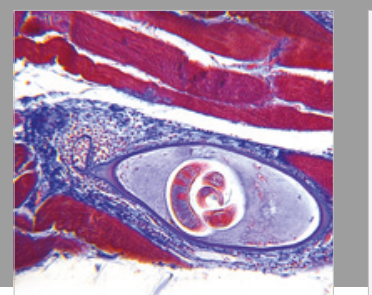

Gastroenterology Research and Practice

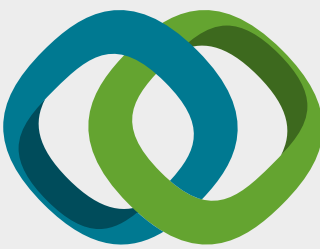

\section{Hindawi}

Submit your manuscripts at

www.hindawi.com
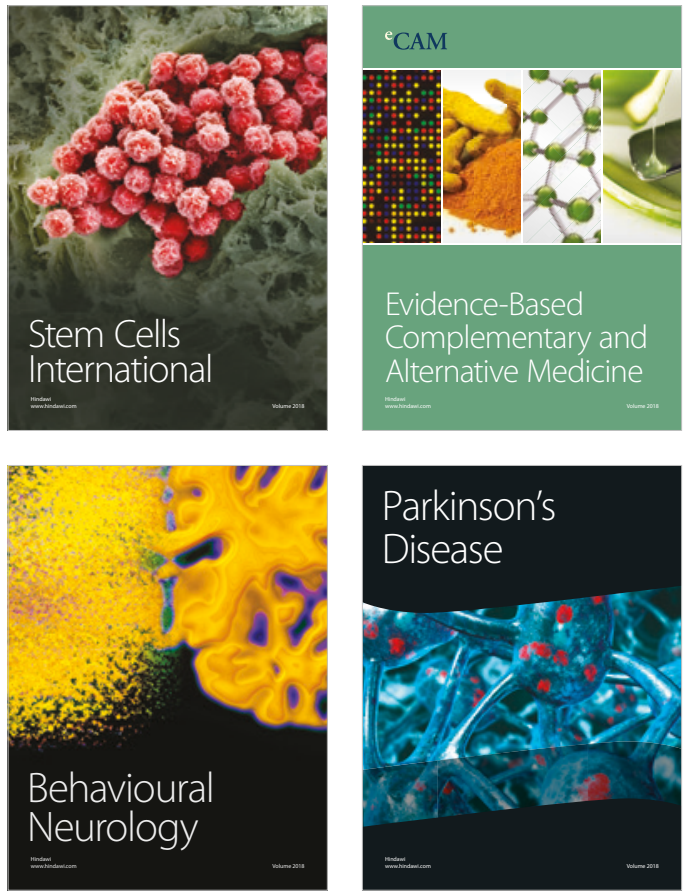

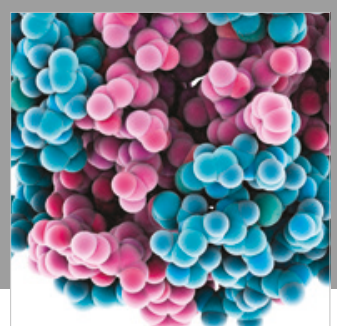

ournal of

Diabetes Research

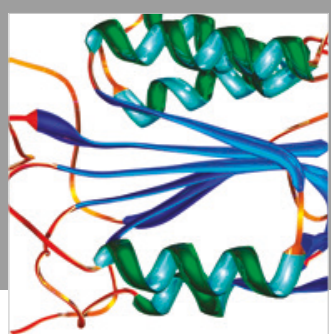

Disease Markers
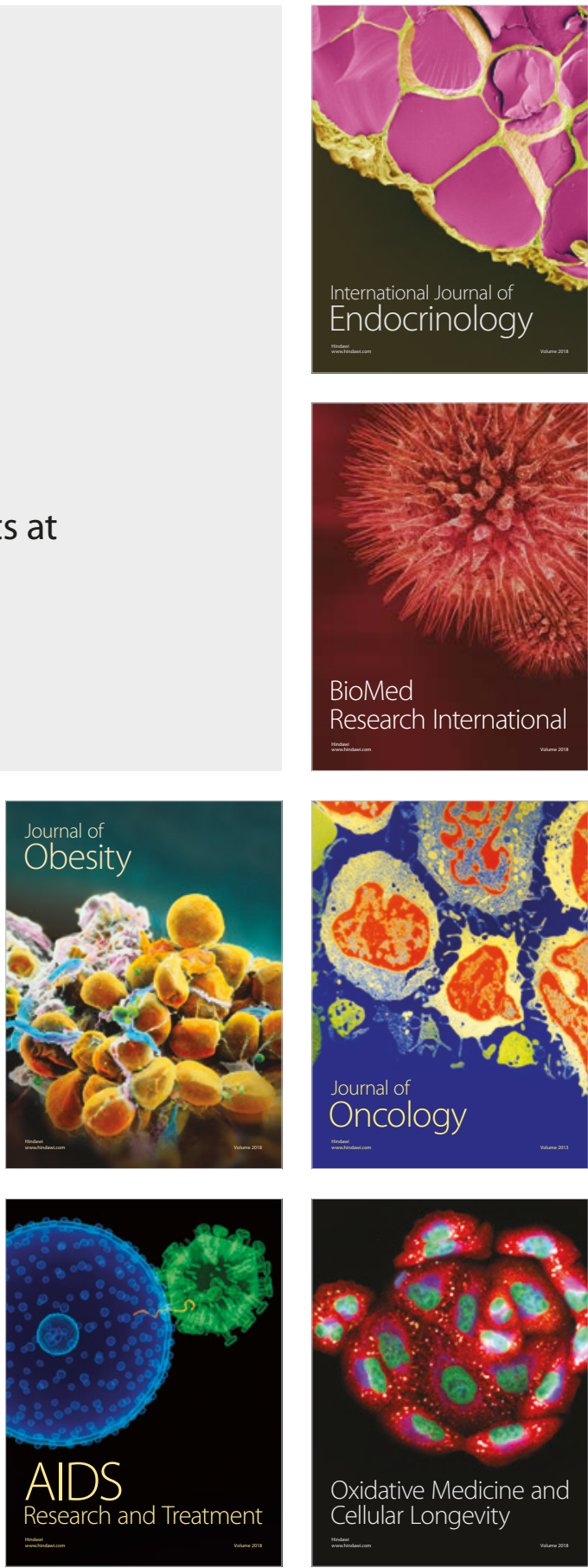